\title{
EXPRESSION OF TRANSCRIPTION FACTOR COUP-TF1 (NR2F1) IN DEVELOPING OCCIPITAL CORTEX IN HUMANS
}

\author{
Meglena Angelova ${ }^{1}$, Radoslav Minkov ${ }^{2}$, Vanya Goranova ${ }^{1}$, Stoyan Pavlov ${ }^{1}$, Vesselina \\ Michaleva ${ }^{1}$, Anton B. Tonchev ${ }^{1}$ \\ ${ }^{1}$ Department of Anatomy, Histology and Embryology, Medical University of Varna, \\ Bulgaria, ${ }^{2}$ University Hospital "Prof. Stamatov" - Varna, Bulgaria
}

\begin{abstract}
PURPOSES: We aimed to investigate the presence, distribution and abundance of chicken ovalbumin upstream promoter transcription factor 1 (COUP-TF1; also known as Nuclear Receptor Subfamily 2 Group F Members 1/2 - NR2F1) in the germinative zones of human telencephalon during the fetal period. This transcription factor is of significance for the normal neuronal migration and differentiation of projection neurons in the mouse forebrain.

MATERIALS AND METHODS: Brain tissue samples from spontaneously aborted human fetuses aged between 12 and 28 gestational weeks (g.w.) were fixed in paraformaldehyde and histologically investigated. Sections immunostained for COUP-TF1 were scanned and its expression in different zones of the occipital lobe of developing pallium was evaluated. COUP-TF1 positive cells were counted and their percentage of all DAPI-stained nuclei was calculated in order to establish the abundance of the COUP-TF1-expressing cells among the total cell population.

RESULTS: COUP-TF1 expression was prominent in all investigated zones of the developing human occipital lobe at stage $17^{\text {th }}$ g.w. In all zones studied, COUP-TF1 positive cells presented a relatively high fraction $(\sim 71-95 \%)$ of the cells counted with the highest value of $95.24 \%$ in the ventricular zone.

CONCLUSIONS: The present data on the location, abundance and distribution pattern of COUP-TF1-expressing cells in the human occipital lobe provide information on the possibility that this TF might participate to human corticogenesis, similarly to the mouse.
\end{abstract}

Keywords: COUP-TF1, NR2F1, cortical progenitors, human fetal telencephalon

Address for correspondence:

Anton Tonchev

Department of Anatomy, Histology and Embryology

Medical University of Varna

55 M. Drinov Str

9002 Varna, Bulgaria

e-mail: anton.tonchev@mu-varna.bg

phone: +35952677082

Received: January 29, 2015

Accepted: March 11, 2015

\section{INTRODUCTION}

The cerebral cortex of mammals is generated predominantly during the embryonic period by stem cells and their derivative progenitors in the pallium of the developing telencephalon. Various genes in complex interactions are involved in the processes of differentiation of the cerebral cortical neurons $(1,2)$. Chicken ovalbumin upstream promoter transcription factors (COUP-TFs) also designated as Nuclear Receptor Subfamily 2 Group F Members 1/2 
(NR2F1/NR2F2) are orphan members of the steroid/thyroid hormone receptor superfamily. COUPTF genes have been cloned from many species and their sequences are exceptionally conserved through evolution. This suggests a critical role for the COUPTFs in these organisms. They play a key role in the regulation of organogenesis, neurogenesis, and cellular differentiation during prenatal development (35). COUP-TFs are also involved in the regulation of several genes that encode metabolic enzymes. They have been shown to negatively regulate the activation function of vitamin $\mathrm{D}$, thyroid hormone, retinoic acid, the retinoid $\mathrm{X}$ and the peroxisome proliferatoractivated receptors $(6,7)$. COUP-TF1 (NR2F1) has been shown to interact with other transcription factors such as Oct-3/4, Sp8, Pax6, Emx2 and Fgf signaling pathway (8-10). It modulates the migration of supragranular cortical neurons and favors proper differentiation of projection neurons (11). COUP-TF1 negatively regulates the expression of TF Pax6 (12), a key regulator of cortical development. A low-rostral to high-caudal gradient is described for COUP-TF1 and its ablation in cortical progenitors causes transformation of more caudally located primary sensory areas into the rostrally located frontal/motor areas.

The aim of the present study was to investigate the expression and distribution of COUP-TF1 in various regions of developing human cortex during the embryonic period.

\section{MATERIAL AND METHODS}

Tissue samples from spontaneously aborted human fetuses aged 12 to 28 gestational weeks (g.w.) were acquired with an informed written consent of the patient (mother) and in strict observance of the current legal and ethical regulations (approved research protocol № 19/05.04.2012 of the Research Ethic Committee at Medical University of Varna). The tissues were fixed in 4\% paraformaldehyde for 2 weeks, and subsequently processed for paraffin or frozen sections and stained by standard histological and immunohistochemical techniques. Sets of paraffin sections were stained by cresyl violet for overview and orientation of the zones and layers. Sets of cryosections were stained by indirect immunofluorescence for COUP-TF1/NR2F1. The mouse primary antibody IgG2a, dilution 1:100 (Perseus Proteomics, Tokyo, Japan) was applied at $4^{\circ} \mathrm{C}$ overnight. The sec- ondary anti-mouse antibody (conjugated to Alexa Fluor-488, Invitrogen), dilution 1:200 was applied for 2 hours at room temperature. Cell nuclei were stained in blue with DAPI. Images were acquired with Axio Imager.72 Zeiss using AxioCam MRc and AxioVisioner software (Carl Zeiss Microscopy). COUP-TF1 positive cells were counted in different zones of the occipital lobe of $17^{\text {th }}$ g.w. human fetal telencephalon and then calculated as a percentage of all DAPIstained nuclei.

\section{RESULTS}

Specific cortical zones of the developing human fetal telencephalon were identified and investigated on sections stained with cresyl violet (Fig. 1).

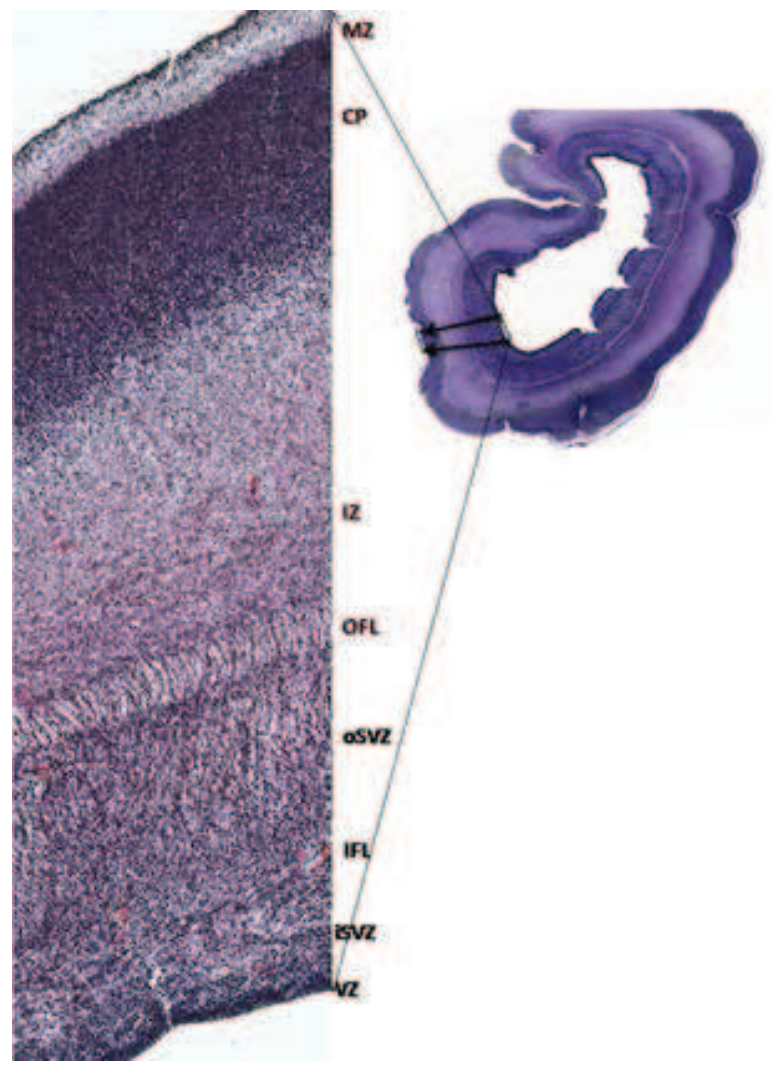

Fig. 1. Germinal zones in the occipital lobe of human fetal telencephalon at $17^{\text {th }}$ g.w.; VZ- ventricular zone; iSVZ inner subventricular zone; IFL - inner fibre layer; oSVZ - outer subventricular zone; OFL - outer fibre layer; IZ - intermediate zone; $C P$ - cortical plate; $M Z$ - marginal zone. Cresyl violet staining (magnifications $x 400,40$ ) 
Meglena Angelova, Radoslav Minkov, Vanya Goranova et al.

COUP-TF1 expression in the occipital cortex at stage $17^{\text {th }}$ g.w. was detected in all major zones of the pallium, including the ventricular zone (VZ), the outer subventricular zone (oSVZ), the intermediate zone (IZ), the cortical plate (CP), and the marginal zone (MZ). These are zones where cortical progenitors are born (VZ/SVZ) and migrate through IZ toward their journey toward the $\mathrm{CP}$ during development (Fig. 1).

In order to evaluate the abundance of the COUP-TF1 positive cells, we compared their number with the number of all cells (DAPI-stained) (Fig. 2).

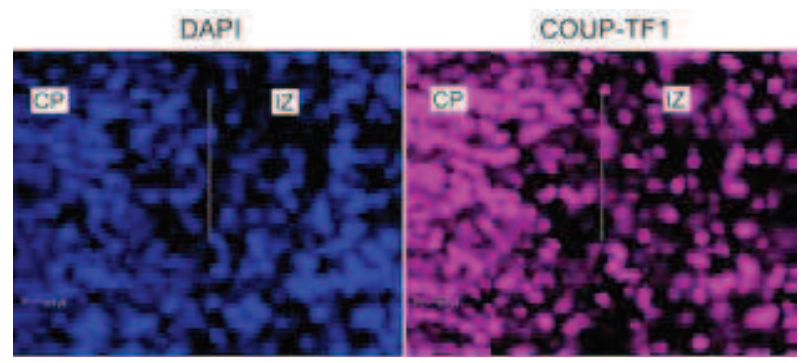

Fig. 2. Fluorescence labeling of cell nuclei with DAPI (blue) and COUP-TF1 (magenta) in the occipital lobe of human fetal telencephalon during $17^{\text {th }} g$. $w$.; $C P$, cortical plate; IZ, intermediate zone

In all zones investigated the COUP-TF1 expressing cells presented a relatively high fraction $(\sim 71-$ $95 \%)$ of the cells with the highest value of $95.24 \%$ detected in the VZ (Fig. 3).

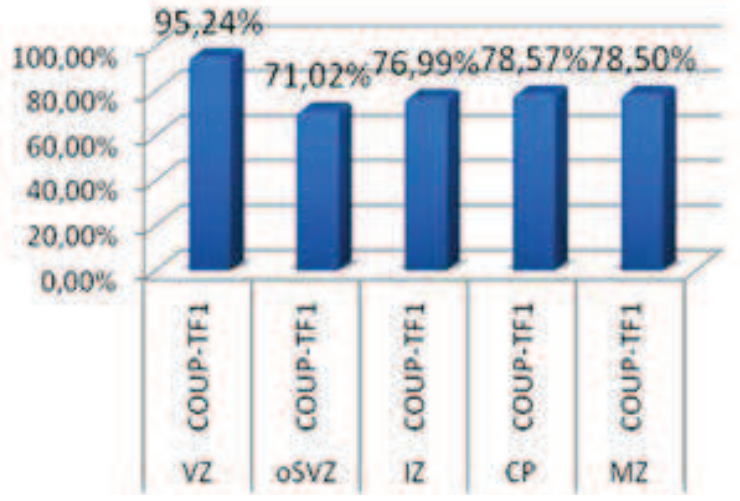

Fig. 3. Percentages of COUP-TF1 positive cells in the

different zones of the occipital lobe of human fetal telencephalon during $17^{\text {th }}$ g.w.; VZ - ventricular zone; oSVZ - outer subventricular zone; IZ - intermediate zone; $C P$ - cortical plate; $M Z$ - marginal zone

\section{DISCUSSION}

Here we show that TF COUP-TF1/NR2F1 is expressed by cortical progenitors and immature neurons in developing occipital cortex of humans at the early stages of cortical neurogenesis (g.w. 17). Further, we estimate that nearly all progenitors in $\mathrm{VZ}$ and the majority of oSVZ progenitors and CP cells as well as cells in the IZ express COUP-TF1/NR2F1. In mice, COUP-TF1 is a critical regulator of cortical regionalization and the maintenance of cortical identity (9). Analyses of null mice showed a misbalance between early-born and late-born cortical neurons, which is consistent with our finding of massive COUP-TF1 expression in early born human neocortical progenitors. Further, miswired area-specific connections between the cortex and the thalamus in COUP-TF1 null mice indicate this TF plays a critical role in the establishment of proper neocortical connections $(13,14)$. Our data on COUP-TF1 expression are in consent with previous findings that this transcription factor is a key promoter of caudal cortical identity (10). Disturbances in COUP-TF1 guided events related to neuronal cortical progenitors may be implicated in human neocortical disorders.

\section{CONCLUSIONS}

COUP-TF1 expression is abundant in cortical progenitors and immature neurons of the developing human occipital lobe. Thus, COUP-TF1 might be involved in the regulation of human neocortical neurogenesis, similarly to its function in the mouse $(9-11,13,14)$.

\section{ACKNOWLEDGEMENTS}

Research was supported by a grant from the local science fund of the Medical University, Varna (№ 14010/18.12.2014).

\section{REFERENCES}

1. Ragsdale CW, Grove EA. Patterning the mammalian cerebral cortex. Curr Opin Neurobiol. 2001;11:50-8.

2. MuhChyi C, Juliandi B, Matsuda T, Nakashima K. Epigenetic regulation of neural stem cell fate during corticogenesis. Int J Dev Neurosci. 2013;31(6):424-33.

3. Sugiyama T, Wang JC, Scott DK, Granner DK. Transcription activation by the orphan nuclear receptor, chicken ovalbumin upstream promoter- 
transcription factor I (COUP-TFI). Definition of the domain involved in the glucocorticoid response of the phosphoenolpyruvate carboxykinase gene. J Biol Chem. 2000;275(5):3446-54.

4. Yamaguchi H, Zhou C, Lin SC, Durand B, Tsai SY, Tsai MJ. The nuclear orphan receptor COUP-TFI is important for differentiation of oligodendrocytes. Dev Biol. 2004;266(2):238-51.

5. Wang L, Shiraki A, Itahashi M, Akane H, Abe H, Mitsumori K et al. Aberration in epigenetic gene regulation in hippocampal neurogenesis by developmental exposure to manganese chloride in mice. Toxicol Sci. 2013;136(1):154-65.

6. Pereira FA, Tsai MJ, Tsai SY. COUP-TF orphan nuclear receptors in development and differentiation. Cell Mol Life Sci. 2000;57(10):1388-98.

7. Zhuang Y, Gudas LJ. Overexpression of COUP-TF1 in murine embryonic stem cells reduces retinoic acid-associated growth arrest and increases extraembryonic endoderm gene expression. Differentiation. 2008;76(7):760-71.

8. Ben-Shushan E, Sharir H, Pikarsky E, Bergman Y. A dynamic balance between ARP-1/ COUP-TFII, EAR-3/COUP-TFI, and retinoic acid receptor:retinoid $\mathrm{X}$ receptor heterodimers regulates Oct-3/4 expression in embryonal carcinoma cells. Mol Cell Biol. 1995;15(2):1034-48.

9. Zhou C, Tsai SY, Tsai MJ. COUP-TFI: an intrinsic factor for early regionalization of the neocortex. Genes Dev. 2001;15(16):2054-9.

10. 1Borello U, Madhavan M, Vilinsky I, Faedo A, Pierani A, Rubenstein J et al. Sp8 and COUPTF1 reciprocally regulate patterning and Fgf signaling in cortical progenitors. Cereb Cortex. 2014;24(6):1409-21.

11. Alfano C, Viola L, Heng JI, Pirozzi M, Clarkson M, Flore G, et al. COUP-TFI promotes radial migration and proper morphology of callosal projection neurons by repressing Rnd2 expression. Development. 2011;138(21):4685-97.

12. Piñon MC, Tuoc TC, Ashery-Padan R, Molnár Z, Stoykova A. Altered molecular regionalization and normal thalamocortical connections in cortex-specific Pax6 knock-out mice. J Neurosci. 2008;28(35):8724-34.

13. Armentano M, Chou SJ, Tomassy GS, Leingärtner A, O'Leary DDM, Studer M. COUP-TFI regulates the balance of cortical patterning between frontal/motor and sensory areas. Nat Neurosci. 2007;10:1277-86.

14. Faedo A, Tomassy GS, Ruan Y, Teichmann H, Krauss S, Pleasure SJ et al. COUP-TF1 coordinates cortical patterning, neurogenesis, and laminar fate and modulates MAPK/ERK, AKT and betacatenin signaling. Cereb Cortex. 2008;18(9):2117-31. 